\title{
STATE ESTIMATION FOR MISO NON-LINEAR SYSTEMS IN CONTROLLER CANONICAL FORM
}

\author{
BENOÎT SCHWALLER $^{a}, *$, DENIS ENSMINGER ${ }^{a}$, BIRGITTA DRESP-LANGLEY $^{b}$, JOSÉ RAGOT $^{c}$
}

\author{
${ }^{a}$ Laboratory of Inventive Design (LGECO), EA 3938 INSA Strasbourg \\ University of Strasbourg, 24 Boulevard de la Victoire, 67000 Strasbourg, France \\ e-mail: schwaller@convergence.u-strasbg.fr \\ ${ }^{b}$ Laboratory of Engineering, Informatics and Imaging (ICUBE) \\ University of Strasbourg, UMR 7357 CNRS, 2 Rue Boussingault, 67000 Strasbourg, France \\ e-mail: birgitta.dresp@unistra.fr \\ ${ }^{c}$ Nancy Research Center in Automatic (CRAN), UMR 7039 \\ University of Lorraine, CNRS, 2 Avenue de Haye, 54516 Vandœuvre lès Nancy, France \\ e-mail: Jose.Ragoteensem.inpl-nancy.fr
}

\begin{abstract}
We propose a new observer where the model, decomposed in generalized canonical form of regulation described by Fliess, is dissociated from the part assuring error correction. The obtained stable exact estimates give direct access to state variables in the form of successive derivatives. The dynamic response of the observer converges exponentially, as long as the nonlinearities are locally of Lipschitz type. In this case, we demonstrate that a quadratic Lyapunov function provides a number of inequalities which guarantee at least local stability. A synthesis of gains is proposed, independent of the observation time scale. Simulations of a Düffing system and a Lorenz strange attractor illustrate theoretical developments.
\end{abstract}

Keywords: non-linear systems, state observers, continuous time.

\section{Introduction}

State observers have been intensely exploited since Luenberger (1966) to model, control or identify linear and non-linear systems, including the studies of Krener and Isidori (1983), Zeitz (1987) or Zheng et al. (2009), related to non-linear systems transformable into canonical form. The key idea in such approaches is to produce approximate measures of non-linearity of order 1, as in extended Luenberger observers (ELOs) (Ciccarella et al., 1993). Approximations of non-linearities in canonical form (which results in an ELO) have already been suggested by Bestle and Zeitz (1983), and this approach can be extended to higher order approximations (Röbenack and Lynch, 2004). An observer using partial non-linear observer canonical form (POCF) (Röbenack and Lynch, 2006) has weaker observability and integrability

\footnotetext{
* Corresponding author
}

existence conditions than the well-established non-linear observer canonical form (OCF). Non-linear sliding mode observers use a quasi-Newtonian approach, applied after pseudo-derivations of the output signal (Veluvolu et al., 2007; Efimov and Fridman, 2011). State observers using extended Kalman filters (EKFs) provide another method of transforming non-linear systems (Boker and Khalil, 2013; Rauh et al., 2013). Finding an appropriate method for parameter synthesis remains one of the major difficulties with state observers for non-linear systems. Tornambè (1992), Farza et al. (2011) and Mobki et al. (2015) proposed high-gain state observers to deal with this problem. High-gain state observers reduce observation errors for a range of predetermined amplitudes or fluctuations by making the observations independent of parameters. The weak point of this method is its sensitivity to noise and uncertainty.

In network identification and encryption, observers with delays are used to synchronize chaotic oscillators, 
as shown in several studies (Ghosh et al., 2010; Martínez-Guerra et al., 2011). Noise and uncertainty are not critical factors in such a context. This can be very different in the case of industrial processes, as shown in a recent study by Bodizs et al. (2011), where the performances of observers using an ELO, EKF or integrated Kalman filters (IKFs) are compared. The influence of noise and uncertainty on these observer types was emphasized, with more reliable results produced by ELOs, which permit exact state reconstruction of highly perturbed systems. For PI and ELO observer classes, Söffker et al. (1995) as well as Morales and Ramirez (2002) demonstrated a compensation effect on measurement errors. Chen et al. (2011) and Bouraoui et al. (2015) addressed the problem of uncertainty of non-linear models. One way of overcoming the problem of parametric uncertainty is to use adaptive observers (Tyukina et al., 2013; Alma and Darouach, 2014; Farza et al., 2014), in the particular case where the measurements are only available at discrete instants and have disturbances. Another approach (Mazenc and Dinh, 2014; Thabet et al., 2014) consists in defining interval observers. Modeling observer systems by Takagi-Sugeno decomposition (Bezzaoucha et al., 2013; Guerra et al., 2015 ) is another possibility, as is the use of models with symmetries and semi-invariants (Menini and Tornambè, 2011).

In a precedent study (Schwaller et al., 2013), we dealt with a specific class of non-linear SISO (single input single output) systems, described by Fliess (1990), called the generalized controller canonical form (Zeitz, 1985). In principle, every uniformly observable (Hermann and Krener, 1977; Gauthier and Bornard, 1981) smooth enough SISO system with vector input $\underline{u}(t)$ and output $y(t)$ can be transformed into this normal form, and extended to the following MISO (multiple input single output) systems (Glumineau and Lôpez-Morales, 1999):

$$
\begin{aligned}
& \underline{\dot{x}}(t)=\underline{A} \underline{x}(t)+\underline{f}(t), \\
& y(t)=\underline{c}^{T} \underline{x}(t)+\Phi[\underline{U}(t)], \\
& \underline{A}=\delta_{i j}, \quad j=i+1, \quad i=1, \ldots, n-1, \\
& \underline{c}^{T}=\left[\begin{array}{lll}
c_{1} & \ldots & c_{n}
\end{array}\right] \text {, }
\end{aligned}
$$

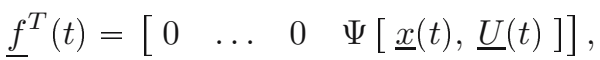

with the following definitions:

$n$ : order of differential equation,

$m$ : number of independent inputs,

$u_{j i}(t):(i-1)$-th temporal derivative of the input $j$ $\left(u_{j 1}(t) \ldots u_{j i}(t)\right)$,

$\underline{u}_{i}(t)^{T}$ : vector $\left[u_{1 i}(t), \ldots u_{m i}(t)\right]$ of $m$ derivatives of degree $i-1$ of inputs,

$\underline{U}(t): n \times m$ input matrix,

$x_{i}(t):(i-1)$-th temporal derivative of $x_{1}(t)$,
$\underline{x}^{T}(t):$ state vector $\left[x_{1}(t), \ldots x_{n}(t)\right]$,

$\underline{c}$ : vector of model parameters,

$\Psi[\underline{x}(t), \underline{U}(t)]$ : scalar non-linear $C^{1}$ function,

$\Phi[\underline{U}(t)]$ : function of inputs $\underline{U}(t)$,

$y(t)$ : output variable,

$\theta \leqslant n$ : index of last coefficient $c_{i} \neq 0$,

$\underline{A}: n \times n$ matrix whose last line is zero.

We previously limited the field of application of the proposed observer because of the decomposition of the function $\Psi(t)$ into two distinct parts which had the effect of limiting the functions $\Psi(t)$ to the third order, and necessitated the integration of all or part of the latter. It can also be noted that the proposed limiting conditions of stability could prove restrictive, above all for second order systems, because of the big increase of the Lipschitz constant in such cases. We propose here to overcome these difficulties by modifying the observer structure, and by scaling differently the differential equation of the physical system. This will have the effect of relaxing the limiting stability conditions of the observer and to increase the field of application, to cover the same domain as that proposed by Gauthier et al. (1992), extended to MISO systems described by (1). The proposed approach is completely deterministic and the only requirement is that the non-linear functions be at least $C^{1}$, and their time derivatives $\mathrm{d} \Psi[\underline{x}(t), \underline{U}(t)] / \mathrm{d} t$ be globally Lipschitz in $\underline{x}(t)$ (Raghavan and Hedrick, 1994). This assumption can be relaxed so that the derivatives are only locally Lipschitz, or can be transformed adequately, for many practical applications (Düffing, van der Pol, Bernoulli equations, inverted penduli, non-linear friction models for DC motors or valve actuators, bioreactors, strange attractors, etc.).

The second aim is to dissociate the state estimations used to reconstruct the functions $\Psi(t)$ of error corrections, in order to increase their insensitivity to noise and to counteract this well-known fault of high gain observers. Conserving the structure PI of the precedent observer, one models the external perturbations of the model and keeps a unity static gain to the dynamics of the convergence of the ensemble.

Let us fix at present the cut-off frequency $1 / T_{o}$ of the observer, and also the pulse $\omega_{o}=2 \pi / T_{o}$, in order to transform the representation of the state of the physical system. This has the considerable advantage of giving a normalized space that is independent of the temporal dynamics of the system, allowing in Section 2.3 to control the conditions of stability of the observer and presenting in Section 2.4 a systematic algebraic approach for the synthesis of the gains of the observer.

Definition 1. This gives the following scaled state representation:

$$
\underline{\dot{x}}(\tau)=\underline{A} \underline{x}(\tau)+\underline{f}(\tau),
$$




$$
\begin{gathered}
y(\tau)=\underline{\tilde{c}}^{T} \underline{x}(\tau)+\Phi[\underline{U}(\tau)], \\
\underline{\tilde{c}}^{T}=\left[\begin{array}{lll}
\widetilde{c}_{1} & \ldots & \widetilde{c}_{n}
\end{array}\right], \\
\underline{f}(\tau)^{T}=\left[\begin{array}{llll}
0 & \ldots & 0 & \widetilde{\Psi}[\underline{x}(\tau), \underline{U}(\tau)]
\end{array}\right],
\end{gathered}
$$

with

$$
\begin{array}{rlrl}
\tau & =\omega_{o} t, & \dot{x}_{n}(t) & =\dot{x}_{n}(\tau) \omega_{o}^{n}, \\
u_{i j}(t) & =u_{i j}(\tau) \omega_{o}^{i-1}, & x_{i}(t) & =x_{i}(\tau) \omega_{o}^{i-1}, \\
\widetilde{c}_{i}=c_{i} \omega_{o}{ }^{i-1}, & i & =1, \ldots, n .
\end{array}
$$

Such a normalized representation is possible in time (Gille et al., 1988) as well as in the frequency domain (Gißler and Schmid, 1990) for linear systems.

$f(\tau)$ is a vector of dimension $n$. Equations (3) define time dilation or retraction of the state representation and its new parameters, without changing the pattern of the signal $x_{i}(\tau)$. For the function $\Psi$, this is translated by the relation of changing the following scale representation:

$$
\Psi[\underline{x}(t), \underline{U}(t)]=\omega_{o}^{n} \tilde{\Psi}[\underline{x}(\tau), \underline{U}(\tau)] .
$$

The function $\widetilde{\Psi}[\underline{x}(\tau), \underline{U}(\tau)]$ is obtained by replacing every state or command variable by the corresponding one in (3) and dividing everything by $\omega_{o}{ }^{n}$ (an example given in Section 3, cf. (58) and (60)). In the rest of the study, in Section 2.1 we define the observer structure and the role of its different components. In Section 2.2 we study its dynamics and search for the differential equation defining the physical-observer system state distances. In Section 2.3 we demonstrate the stability of the state estimates and the exponentially convergent character of the estimates. In Section 2.4 we obtain the synthesis of the gains of the observer and exploit the transformation defined in (2). Finally, in Section 3, two different simulations illustrate the developments of Section 2 .

\section{Structure and synthesis of the observer}

2.1. System and observer definitions. To begin with, let us isolate the component $x_{1}(\tau)$ of (2b), which will subsequently serve to determine the observation error. To obtain $y_{1}(\tau)$, the estimation of variable $x_{1}(\tau)$, three cases are distinguished.

For $\theta=1$, we have

$$
y_{1}(\tau)=\frac{y(\tau)-\Phi[\underline{U}(\tau)]}{\widetilde{c}_{1}} .
$$

For $\theta=2$, it becomes

$$
\dot{y}_{1}(\tau)=-\frac{\widetilde{c}_{1}}{\widetilde{c}_{2}} y_{1}(\tau)+\frac{y(\tau)-\Phi[\underline{U}(\tau)]}{\widetilde{c}_{2}} .
$$

In the most general case, where $\theta>2, y(\tau)-\Phi[\underline{U}(\tau)]$ is filtered by

$$
\begin{aligned}
& \underline{\dot{w}}(\tau)=\underline{K} \underline{w}(\tau)+\underline{k}[y(\tau)-\Phi[\underline{U}(\tau)]], \\
& \underline{K}=\left[\begin{array}{cccc}
0 & 1 & 0 & \ldots \\
0 & 0 & 1 & \ldots \\
\ldots & 0 & 0 & 1 \\
-\frac{\widetilde{c}_{1}}{\widetilde{c}_{\theta}} & \ldots & \ldots & -\frac{\widetilde{c}_{\theta-1}}{\widetilde{c}_{\theta}}
\end{array}\right], \\
& \underline{w}(\tau)^{T}=\left[\begin{array}{lll}
y_{1}(\tau) & \ldots & y_{\theta-1}(\tau)
\end{array}\right], \quad \underline{w}(0)=\underline{0}, \\
& \underline{k}^{T}=\left[\begin{array}{llll}
0 & \ldots & 0 & 1 / \widetilde{c}_{\theta}
\end{array}\right] .
\end{aligned}
$$

To analyze the effect of the filter, we rewrite (2b) in scalar form, ignoring $\widetilde{c}_{\theta+1} \ldots \widetilde{c}_{n}$, which are all zero,

$$
\frac{y(\tau)-\Phi[\underline{U}(\tau)]}{\widetilde{c}_{\theta}}=x_{\theta}(\tau)+\sum_{i=1}^{\theta-1} \frac{\widetilde{c}_{i}}{\widetilde{c}_{\theta}} x_{i}(\tau)
$$

Substituting (8) in the $(\theta-1)$-th component of $(7 \mathrm{a})$, we get

$$
\widetilde{c}_{\theta} \dot{y}_{\theta-1}(\tau)+\sum_{i=1}^{\theta-1} \widetilde{c}_{i} y_{i}(\tau)=\sum_{i=1}^{\theta} \widetilde{c}_{i} x_{i}(\tau)
$$

whose Laplace transform gives

$$
\begin{aligned}
& y_{1}(s)\left[\widetilde{c}_{1}+\ldots \widetilde{c}_{\theta} s^{\theta-1}\right] \\
&=x_{1}(s)\left[\widetilde{c}_{1}+\ldots \widetilde{c}_{\theta} s^{\theta-1}\right] .
\end{aligned}
$$

The transfer function $y_{1}(s) / x_{1}(s)$ is equal to 1 ; then $x_{1}(\tau)=y_{1}(\tau)$, and more generally $x_{i}(\tau)=y_{i}(\tau)$ for $i=$ $1, \ldots, \theta$. In practice, if $y(t)$ is noisy, only $y_{1}(t)$ will be really usable and will serve to determine the observation error.

Definition 2. To generate state estimates $\underline{v}(\tau)$ for the system (2), a PI observer structure is defined with

$$
\begin{aligned}
& \underline{\dot{\check{x}}}(\tau)=\underline{A} \underline{\breve{x}}(\tau)+\underline{\tilde{f}}(\tau)+\underline{\breve{h}} \Delta y_{1}(\tau), \\
& \underline{\dot{\hat{x}}}(\tau)=\underline{A} \underline{\hat{x}}(\tau)+\underline{\breve{A}} \underline{\breve{x}}(\tau)+\underline{\widehat{h}} \Delta y_{1}(\tau), \\
& \Delta y_{1}(\tau)=x_{1}(\tau)-\widehat{x}_{1}(\tau) \text {, } \\
& \underline{\tilde{f}}(\tau)^{T}=\left[\begin{array}{llll}
0 & \ldots & 0 & \tilde{f}(\tau)
\end{array}\right], \\
& \dot{I}_{0}(\tau)=h_{0} \Delta y_{1}(\tau) \text {, } \\
& \tilde{f}(\tau)=I_{0}(\tau)+\widetilde{\Psi}[\underline{v}(\tau), \underline{U}(\tau)],
\end{aligned}
$$




$$
\begin{aligned}
& \underline{\check{x}}(\tau)^{T}=\left[\begin{array}{lll}
\breve{x}_{2}(\tau) & \ldots & \breve{x}_{n}(\tau)
\end{array}\right], \\
& \underline{\widehat{x}}(\tau)^{T}=\left[\begin{array}{lll}
\widehat{x}_{1}(\tau) & \ldots & \widehat{x}_{n-1}(\tau)
\end{array}\right], \\
& \underline{v}(\tau)^{T}=\left[\begin{array}{ll}
\widehat{x}_{1}(\tau) & \underline{\check{x}}(\tau)^{T}
\end{array}\right] \\
& \underline{\hat{x}}(0)=\underline{\check{x}}(0)=\underline{0}, \quad I_{0}(0)=0, \\
& \underline{\check{h}}^{T}=\left[\begin{array}{ll}
\underline{0}^{T} & h_{1}
\end{array}\right], \\
& \underline{\hat{h}}^{T}=\left[\begin{array}{lll}
h_{n} & \ldots & h_{2}
\end{array}\right] \text {, } \\
& \underline{h}^{T}=\left[\underline{\hat{h}}^{T}, h_{1}\right] \text {, } \\
& \underline{A}=\delta_{i j}, \quad j=i+1, i=1, \ldots, n-1, \\
& \underline{\widetilde{A}}=\left[\begin{array}{cccc}
0 & \ldots & \ldots & 0 \\
\vdots & \ddots & \ldots & \vdots \\
\vdots & & 0 & 0 \\
0 & \ldots & 0 & 1
\end{array}\right] \text {, }
\end{aligned}
$$

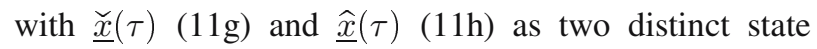
vectors of dimension $n-1$, coupled using the matrices $\underline{A}$ (11n) and $\underline{A}$ (110 of dimension $(n-1) \times(n-1)$. The vectors $\underline{h}$ and $\underline{h}$ are also of dimension $n-1$. The matrix $\underline{A}$ is constructed using the Kronecker operator, which puts the upper diagonal at 1. Figure1 1 illustrates the functional diagram of such an observer of the third order.

The augmented vector $\underline{v}(\tau)$ (11i), 11h) is used as an estimate of $\underline{x}(\tau)$ and as a variable of the function $\widetilde{\Psi}[\underline{v}(\tau), \underline{U}(\tau)] \underline{11 \mathrm{f})}$. The state $\underline{\hat{x}}(\tau)(11 \mathrm{~b}$ is an observer exploiting the observation error $\Delta y_{1}(\tau)$ (11c) via the gains $h_{i} 11 \mathrm{~m}$, serving to correct the state distances between the system and its observer. In Fig. 1, for example, we have

$$
\begin{aligned}
& \underline{\widehat{x}}(\tau)^{T}=\left[\begin{array}{ll}
\hat{x}_{1}(\tau) & \widehat{x}_{2}(\tau)
\end{array}\right], \\
& \underline{\check{x}}(\tau)^{T}=\left[\begin{array}{ll}
\breve{x}_{2}(\tau) & \breve{x}_{3}(\tau)
\end{array}\right], \\
& \underline{v}(\tau)^{T}=\left[\begin{array}{lll}
\widehat{x}_{1}(\tau) & \breve{x}_{2}(\tau) & \breve{x}_{3}(\tau)
\end{array}\right], \\
& \underline{\hat{h}}^{T}=\left[\begin{array}{ll}
h_{3} & h_{2}
\end{array}\right], \\
& \underline{\breve{h}}^{T}=\left[\begin{array}{ll}
0 & h_{1}
\end{array}\right] \text {. }
\end{aligned}
$$

The choice of using two state variables $\underline{\hat{x}}(\tau)$ and $\underline{\breve{x}}(\tau)$ is motivated by $n-1$ successive integrations of $\dot{\breve{x}}_{n}(\tau)$, in which no re-injection error is involved. This allows an increase in the robustness of the estimates to the measurement noise, which in general affects the variable $y_{1}(\tau)$. One thus overcomes a common weak point of high gain observations, i.e., their sensitivity to measurement noise. The second advantage comes from the non-linear function $\widetilde{\Psi}[\underline{v}(\tau), \underline{U}(\tau)]$, which is no longer subjected to the restrictive conditions used by Schwaller et al. (2013), and covers the ensemble of the systems described by Fliess (1990). The vector $\underline{f}(\tau)$ (11d), of dimension $n-1$, compensates the effects of $f(\tau)$ and of possible external exogenous disturbance of (11) using the integral component $I_{0}(\tau)$ 11e. Note that at the second order, for a gain $h_{0}=0$ inhibiting the integrator $I_{0}$, the observer becomes similar to that proposed by Gauthier et al. (1992) for an SISO system.

2.2. Characterization of the observer error dynamics. Now we try to characterise the dynamics of observations by seeking a differential equation linking the two state variables to the observation error $\Delta y_{1}(\tau)$ and to its successive derivatives. The error $\Delta y_{1}(\tau)$ and its successive time derivatives, inaccessible to the measurement, are the state vector of system/observer errors. We want to ultimately determine the state equations of these errors. We will introduce all the necessary notation to put it in the matrix form of the observation error. To this end, we deduce from (11b) the recursive relation used to generate estimates for successive state characteristics $\widehat{x}_{i}(\tau)$ as functions of the output errors:

$$
\begin{aligned}
\widehat{x}_{i+1}(\tau) & =\dot{\hat{x}}_{i}(\tau)-h_{n-i+1} \Delta y_{1}(\tau), \\
i & =1, \ldots, n-2 .
\end{aligned}
$$

To simplify the expressions for successive derivatives of the observer state $\hat{x}_{1}(\tau)$, we introduce

$$
\underline{\tilde{x}}(\tau)^{T}=\left[\begin{array}{lll}
\tilde{x}_{1}(\tau) & \ldots & \widetilde{x}_{n}(\tau)
\end{array}\right],
$$

whose components are defined by

$$
\begin{aligned}
\widetilde{x}_{i+1}(\tau) & =\frac{\mathrm{d}^{(i)} \widehat{x}_{1}(\tau)}{\mathrm{d} \tau^{(i)}}, \quad i=1, \ldots, n-1, \\
\widetilde{x}_{1}(\tau) & =\widehat{x}_{1}(\tau) .
\end{aligned}
$$

Consequently

$$
\dot{\widetilde{x}}_{i}(\tau)=\widetilde{x}_{i+1}(\tau), \quad i=1, \ldots, n-1 .
$$

Using (14) and (15), it is possible to re-write the observation error $\Delta y_{1}(\tau) \quad[11 \mathrm{c}$ ) and its successive derivatives:

$$
\begin{aligned}
\Delta y_{1}(\tau) & =x_{1}(\tau)-\tilde{x}_{1}(\tau), \\
\Delta y_{i+1}(\tau) & =\frac{\mathrm{d}^{(i)} \Delta y_{1}(\tau)}{\mathrm{d} \tau^{(i)}}, \quad i=1, \ldots, n-1,
\end{aligned}
$$

We deduce from this that

$$
\begin{aligned}
\Delta y_{i+1}(\tau) & =\Delta \dot{y}_{i}(\tau), \quad i=1, \ldots, n-1, \\
\Delta y_{i}(\tau) & =x_{i}(\tau)-\widetilde{x}_{i}(\tau), \quad i=1, \ldots, n, \\
\Delta \underline{y}(\tau)^{T} & =\left[\begin{array}{lll}
\Delta y_{1}(\tau) & \ldots & \Delta y_{n}(\tau)
\end{array}\right] .
\end{aligned}
$$

Now we exploit these new definitions to describe the observer dynamics. We replace (14) in (12) for a given index $i$ given and derive this new relation with respect to time using (15) and 17a). The expression $\dot{\hat{x}}_{i}(\tau)$ thus 


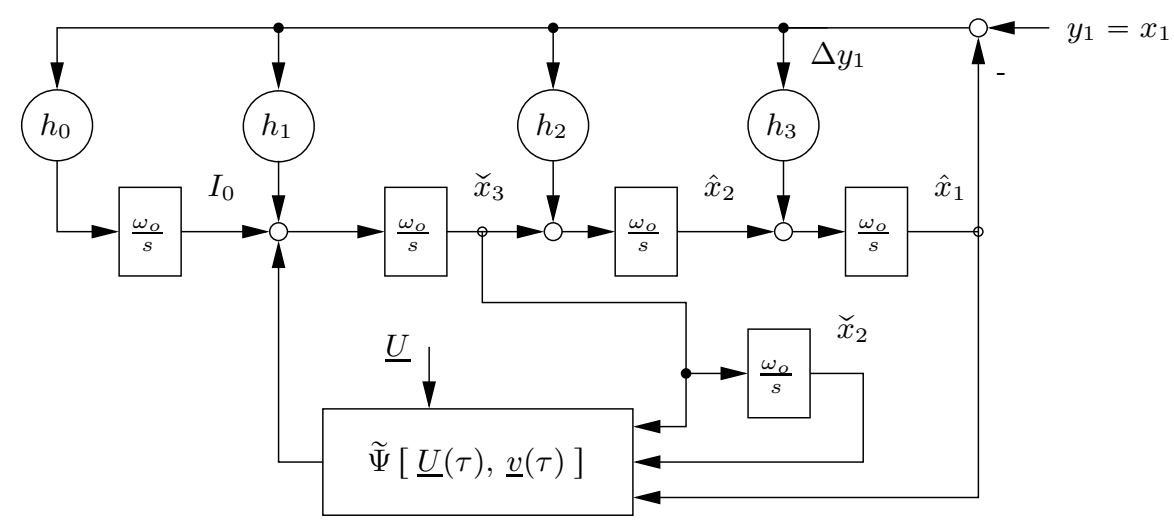

Fig. 1. Third order observer.

obtained may be equated with the same term obtained with (12) for index $i+1$. This operation, carried out successively up to order $n-1$, gives

$$
\dot{\widehat{x}}_{n-1}(\tau)=\tilde{x}_{n}(\tau)-\sum_{i=2}^{n-1} h_{i+1} \Delta y_{i}(\tau) .
$$

Comparing (18) with (11b) to row $n-1$, we obtain

$$
\dot{\hat{x}}_{n-1}(\tau)=\breve{x}_{n}(\tau)+h_{2} \Delta y_{1}(\tau),
$$

which, replaced in (18), gives

$$
\breve{x}_{n}(\tau)=\widetilde{x}_{n}(\tau)-\sum_{i=2}^{n} h_{i} \Delta y_{i-1}(\tau) .
$$

The linear dependence of $\breve{x}_{n}(\tau), \widetilde{x}_{n}(\tau)$ and $\Delta \underline{y}(\tau)$ allows the Laplace transform of (20) and its successive integrations and, because of (15), makes it possible to determine the general form of $\breve{x}_{i}(s)$ :

$$
\begin{aligned}
\check{x}_{i}(s) & =\widetilde{x}_{i}(s)-f_{i}(s), \quad i=2, \ldots, n, \\
f_{i}(s) & =\Delta y_{1}(s) \sum_{j=2}^{n} h_{j} s^{k} \\
k & =i+j-n-2 .
\end{aligned}
$$

The inverse Laplace transform of (21a) is written as

$$
\check{x}_{i}(\tau)=\widetilde{x}_{i}(\tau)-\mathcal{L}^{-1}\left\{f_{i}(s)\right\} .
$$

The second term on the right-hand side of (22) tends towards 0 if the vector $\Delta \underline{y}(\tau) \rightarrow \underline{0}$ as $\tau \rightarrow \infty$.

The derivative of (20) can be compared with that of row $n-1$ from 11a), which gives

$$
\dot{\widetilde{x}}_{n}(\tau)=f(\tau)+\sum_{i=1}^{n} h_{i} \Delta y_{i}(\tau) .
$$

We now try to specify the dynamics of the output distance between the physical system and the observer. To do this, we calculate the distance between the component $n$ of the state vector $\underline{\dot{x}}(\tau)(2 \mathrm{a})$ and $\dot{\widetilde{x}}_{n}(\tau)$ 23). Thus

$$
\begin{aligned}
\Delta \dot{y}_{n}(\tau) & =\dot{x}_{n}(\tau)-\dot{\widetilde{x}}_{n}(\tau) \\
& =\Delta \widetilde{\Psi}(\tau)-I_{0}(\tau)-\sum_{i=1}^{n} h_{i} \Delta y_{i}(\tau),
\end{aligned}
$$

with

$$
\begin{aligned}
\Delta \widetilde{\Psi}(\tau)= & \widetilde{\Psi}[\underline{x}(\tau), \underline{U}(\tau)] \\
& -\widetilde{\Psi}[\underline{\tilde{x}}(\tau), \Delta \underline{y}(\tau), \underline{U}(\tau)] .
\end{aligned}
$$

The distance $\Delta \widetilde{\Psi}(\tau)$ 25, of type $C^{1}$, is obtained from the non-linear functions $\widetilde{\Psi}[\underline{x}(\tau), \underline{U}(\tau)]$ and $\widetilde{\Psi}[\underline{v}(\tau), \underline{U}(\tau)]$ (11f), which are also of type $C^{1}$.

It is now useful to put 24b in matrix form of dimension $n \times n$, using 117a), 24b) and 11d):

$$
\begin{aligned}
\Delta \underline{\dot{y}}(\tau) & =\underline{\tilde{A}} \Delta \underline{y}(\tau)+\Delta \underline{f}(\tau), \\
\Delta \underline{f}(\tau) & =\underline{f}(\tau)-\underline{\tilde{f}}(\tau), \\
\underline{\tilde{A}} & =\left[\begin{array}{cccc}
0 & 1 & 0 & \ldots \\
0 & 0 & 1 & \ldots \\
\ldots & 0 & 0 & 1 \\
-h_{1} & \ldots & -h_{n}
\end{array}\right] .
\end{aligned}
$$

In (26b), the term $\tilde{f}(\tau)$ 11f includes $I_{0}(\tau)$ 11e). This leads to a last derivation on (24b), to obtain the final state representation of the output distances $\Delta y_{1}(\tau)$. For that purpose, it is necessary to define the states and the supplementary distances as follows:

$$
\begin{aligned}
\underline{u}_{n+1}(\tau) & =\underline{\dot{u}}_{n}(\tau), \\
x_{n+1}(\tau) & =\dot{x}_{n}(\tau), \\
\widetilde{x}_{n+1}(\tau) & =\dot{\widetilde{x}}_{n}(\tau), \\
\check{x}_{n+1}(\tau) & =\dot{\check{x}}_{n}(\tau), \\
\Delta y_{n+1}(\tau) & =\Delta \dot{y}_{n}(\tau)
\end{aligned}
$$


to subsequently place the matrix and the augmented vectors:

$$
\begin{aligned}
\underline{U}_{a}(\tau)^{T} & =\left[\begin{array}{ll}
\underline{U}(\tau)^{T} & \underline{u}_{n+1}(\tau)
\end{array}\right], \\
\underline{x}_{a}(\tau)^{T} & =\left[\begin{array}{ll}
\underline{x}(\tau)^{T} & x_{n+1}(\tau)
\end{array}\right], \\
\underline{\tilde{x}}_{a}(\tau)^{T} & =\left[\begin{array}{ll}
\underline{\tilde{x}}(\tau)^{T} & \widetilde{x}_{n+1}(\tau)
\end{array}\right], \\
v_{a}(\tau)^{T} & =\left[\begin{array}{ll}
\underline{v}(\tau)^{T} & \breve{x}_{n+1}(\tau)
\end{array}\right], \\
\Delta \underline{y}_{a}(\tau)^{T} & =\left[\begin{array}{ll}
\Delta \underline{y}(\tau)^{T} & \Delta y_{n+1}(\tau)
\end{array}\right], \\
& =\underline{x}_{a}(\tau)^{T}-\underline{\widetilde{x}}_{a}(\tau)^{T},
\end{aligned}
$$

and separately calculate the time derivative of (25):

$$
\begin{aligned}
\Delta \dot{\Psi}(t)= & \omega_{o}^{n+1} \Delta \dot{\widetilde{\Psi}}(\tau), \\
\Delta \dot{\widetilde{\Psi}}(\tau)= & \dot{\widetilde{\Psi}}\left[\underline{x}_{a}(\tau), \underline{U}_{a}(\tau)\right] \\
& -\dot{\widetilde{\Psi}}\left[\underline{\tilde{x}}_{a}(\tau), \Delta \underline{y}_{a}(\tau), \underline{U}_{a}(\tau)\right] .
\end{aligned}
$$

The time derivative of (24b), using (11e), (17a), (27), (28) and (29), is written in matrix form to obtain the final state representation of the differential equation driving the output error $\Delta y_{1}(\tau)$ :

$$
\begin{aligned}
& \Delta \underline{\dot{y}}_{a}(\tau)=\underline{A}_{a} \Delta \underline{y}_{a}(\tau)+\Delta \underline{\tilde{\Psi}}(\tau),
\end{aligned}
$$

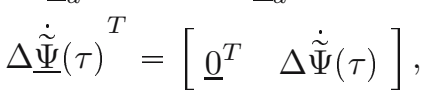

$$
\begin{aligned}
& \underline{A}_{a}=\underline{G}_{a}+\underline{H}_{a}, \\
& \underline{G}_{a}=\delta_{i j}, \quad j=i+1, \quad i=1, \ldots, n, \\
& \underline{H}_{a}^{T}=\left[\begin{array}{llll}
\underline{0} & \cdots & \underline{0} & -\underline{h}_{a}
\end{array}\right], \\
& \underline{h}_{a}^{T}=\left[\begin{array}{lll}
h_{0} & \ldots & h_{n}
\end{array}\right] .
\end{aligned}
$$

In (30d), $\underline{G}_{a}$ is of dimension $(n+1) \times(n+1)$. Its $(n+1)$-th row is zero. The structure of the matrices (30c)-30e makes it possible to deduce the unit static gain of the observer. The system (30) describing the dynamics of convergence of the observation errors is close to that which has previously been proposed (Schwaller et al., 2013), and the conditioning of the system proposed in (3) may be used with advantage.

In 29b and 30b, we assume that $\dot{\widetilde{\Psi}}\left[\underline{x}_{a}(\tau), \underline{U}_{a}(\tau)\right]$ is a non-linear system function Lipschitz in $\underline{x}_{a}(\tau)$ and uniformly bounded in $\underline{U}_{a}(\tau)$ in an invariant set, with a Lipschitz constant $L$, i.e.,

$$
\begin{aligned}
\|\Delta \dot{\tilde{\Psi}}(\tau)\| & \leqslant L\left\|\underline{x}_{a}(\tau)-\underline{\widetilde{x}}_{a}(\tau)\right\| \\
& \leqslant L\left\|\Delta \underline{y}_{a}(\tau)\right\| .
\end{aligned}
$$

Applying the Lipschitz inequality to 29) yields reduction of $\Delta \underline{y}_{a}(\tau)$, the number of useful variables to characterise the perturbing difference $\Delta \dot{\widetilde{\Psi}}(\tau)$. For many systems, if functions $\dot{\widetilde{\Psi}}(\tau)$ are not globally of the Lipschitz type, they can be locally transformed into the Lipschitz type.
2.3. Convergence of state observations. The observer convergence analysis consists in proving the globally asymptotic evolution of the error estimate for state reconstruction. In other words, regardless of the initial conditions, the observer state is to converge toward the state of the physical system. This leads to the following two theorems.

Theorem 1. Let us consider an MISO system decomposable as described in (2), for which the observer structure (11) is used. If the system function $\dot{\widetilde{\Psi}}\left[\underline{x}_{a}(\tau), \underline{U}_{a}(\tau)\right]$ is locally of the Lipschitz type in $\underline{x}_{a}(\tau)$ and uniformly bounded in $\underline{U}_{a}(\tau)$ in an invariant set, with a Lipschitz constant $L$ (31), then the observer (11) will be locally stable if the gains $h_{i}$ in $\underline{A}_{a}$ (30c) can be adjusted so that they satisfy the following conditions:

$$
\begin{aligned}
h_{i} & \geqslant \frac{1}{2 \sigma \phi_{i+1}}+2 \sigma L^{2}\left(\frac{\lambda^{2}}{\phi_{i+1}}+\frac{\phi_{i+1}}{4}\right), \\
h_{n} & \geqslant \frac{1+2 \sigma \phi_{n}}{4 \sigma \phi_{n+1}}+\sigma L^{2}\left(\phi_{n+1}+\sum_{j=1}^{n} \frac{\phi_{j}^{2}}{4 \phi_{n+1}}\right), \\
\lambda & >0, \quad \phi_{i}>0, \quad i=0, \ldots, n-1 .
\end{aligned}
$$

If the system function $\dot{\widetilde{\Psi}}\left[\underline{x}_{a}(\tau), \underline{U}_{a}(\tau)\right]$ is globally of the Lipschitz type and if the gains $h_{i}$ satisfy (32), then the observer (11) will be globally asymptotically stable.

Proof. The proof of Theorem 1 can be achieved by proving the stability of (30a) using an appropriate positive Lyapunov function, like the following quadratic one:

$$
\begin{aligned}
V_{n}(\tau) & =\Delta \underline{y}_{a}(\tau)^{T} \underline{P} \underline{y}_{a}(\tau), \\
\underline{P} & =\left[\begin{array}{cccc}
\lambda & 0 & \ldots & 0 \\
0 & \lambda & \ldots & 0 \\
\vdots & & \ddots & \vdots \\
\phi_{1} & \phi_{2} & \ldots & \phi_{n+1}
\end{array}\right] .
\end{aligned}
$$

$\underline{P}$ is an $(n+1) \times(n+1)$ lower triangular matrix defined as positive and satisfying the Sylvester criteria, with $33 \mathrm{~b}$ ). The proof of convergence is linked to the study of the sign of the derivative of the candidate for a Lyapunov function. This is obtained after time differentiation of $33 \mathrm{a}$, and after substituting (30a) in the result obtained for the terms $\Delta \underline{y}_{a}(\tau)$ :

$$
\begin{aligned}
\dot{V}_{n}(\tau) & =\Delta \underline{y}_{a}(\tau)^{T} \underline{Q} \Delta \underline{y}_{a}(\tau)+N(\tau), \\
\underline{Q} & =\underline{A}_{a}^{T} \underline{P}+\underline{P} \underline{A}_{a}, \\
N(\tau) & =\Delta \underline{y}_{a}(\tau)^{T} \underline{S} \Delta \underline{\tilde{\Psi}}(\tau), \\
\underline{S} & =\underline{P}+\underline{P}^{T} .
\end{aligned}
$$

$N(\tau)$ describes the influence of the non-linear functions on state distances. Calculating the diagonal coefficients 
of (33a), we get a lower triangular matrix $Q$ of dimension $(n+1) \times(n+1)$. The coefficients of the principal diagonal are written as

$$
q_{i i}= \begin{cases}-h_{i-1} \phi_{i}, & i=1, \ldots, n, \\ \phi_{n}-2 \phi_{n+1} h_{n}, & i=n+1 .\end{cases}
$$

If the diagonal coefficients $q_{i i}$ are negative, the Sylvester criterion for semi-negativity is satisfied, and the successive minors of $Q$ will be of opposite signs, ensuring the semi-negativity of the first term on the right-hand side of (34a). Verifying the sign of the second member on the right-hand side of (34a) involves increasing $N(\tau)$ using the Schwartz and Lipschitz inequalities (31b) :

$$
\begin{aligned}
N(\tau) & \leqslant\left\|\Delta \underline{y}_{a}(\tau)^{T} \underline{S} \Delta \underline{\tilde{\tilde{\Psi}}}(\tau)\right\| \\
& \leqslant\left\|\Delta \underline{y}_{a}(\tau)^{T} \underline{S}\right\|\|\Delta \underline{\tilde{\tilde{\Psi}}}(\tau)\| \\
& \leqslant\left\|\Delta \underline{y}_{a}(\tau)^{T} \underline{S}\right\| L\left\|\Delta \underline{y}_{a}(\tau)\right\| .
\end{aligned}
$$

To determine the sign of $\dot{V}_{n}(\tau)$, we apply the inequality:

$$
\begin{aligned}
\left\|\underline{a}(\tau)^{T} \underline{b}(\tau)\right\| & \leqslant \frac{\sigma \underline{a}(\tau)^{T} \underline{a}(\tau)}{2}+\frac{\underline{b}(\tau)^{T} \underline{b}(\tau)}{2 \sigma}, \\
\sigma & >0
\end{aligned}
$$

to $36 \mathrm{c}$ to obtain the desired increase in $N(\tau)$ :

$$
\begin{gathered}
N(\tau) \leqslant \Delta \underline{y}_{a}(\tau)^{T} \underline{R} \Delta \underline{y}_{a}(\tau), \\
\underline{R}=\frac{\sigma L^{2}}{2} \underline{S} \underline{S}+\frac{\underline{I}}{2 \sigma} .
\end{gathered}
$$

Here 38a yields a positive lower triangular matrix $\underline{R}$ (38b) of dimension $(n+1) \times(n+1)$, whose diagonal elements are written as

$$
r_{i i}= \begin{cases}2 \sigma L^{2}\left(\lambda^{2}+\frac{\phi_{i}{ }^{2}}{4}\right)+\frac{1}{2 \sigma}, & i=1, \ldots, n, \\ \sigma L^{2}\left(2{\phi_{i}}^{2}+\frac{1}{2} \sum_{j=1}^{n}{\phi_{j}}^{2}\right)+\frac{1}{2 \sigma}, & i=n+1 .\end{cases}
$$

The inequality (38a) yields 34a):

$$
\begin{aligned}
\dot{V}_{n}(\tau) & \leqslant \Delta \underline{y}_{a}(\tau)^{T} \underline{M} \underline{y}_{a}(\tau), \\
\underline{M} & =\underline{Q}+\underline{R} .
\end{aligned}
$$

With a negative function $\dot{V}_{n}(\tau)$, adding together the diagonal terms (35) and (39), and imposing $Q+\underline{R} \leqslant$ 0 , we obtain the conditions (32). The sum $Q+\underline{R}$ yields a lower triangular matrix that satisfies Sylvester criteria of semi-negativity if the $n$ inequalities (32) are satisfied. Then, if $\Delta \dot{\widetilde{\Psi}}(\tau)$ (25) is Lipschitz (31), $\dot{V}_{n}(\tau)$ is semi-negative and (30a) is globally and asymptotically stable. In (22), it is supposed that the functions $\mathcal{L}^{-1}\left\{f_{i}(s)\right\} \rightarrow 0$ as $t \rightarrow \infty$. Because of this, one can say that $\underline{v}(\tau) \rightarrow \underline{x}(\tau)$ as $t \rightarrow \infty$. The observer is locally stable if (31) is locally Lipschitz.
The stability conditions (32) are less restrictive than those previously proposed (Schwaller et al., 2013). In fact, the pulse $\omega_{0}$ is no longer involved, and the freedom of choice to fix the parameters $\lambda$ and $\phi$ as a function of values that can take the constant of Lipschitz $L$ helps to find a combination where the stability is demonstrated from the gains $h_{i}$ obtained by any method of parameter synthesis. The value of the constant $L$ is generally strongly dependent on the pulse $\omega_{o}$, of the type of non-linearity encountered and of the order $n$ of the physical system.

One way of choosing the parameters $\phi_{i}$ of $\underline{P} 33 \mathrm{~b}$ could be

$$
\phi_{i}=\phi_{n+1}^{n+1-i} .
$$

Thus, the number of parameters to fix in the matrix $\underline{P}$ is limited to $\lambda$ and $\phi_{n+1}$.

Theorem 2. If Theorem 1 can be applied to the system (1) that one wishes to observe, then (11) will be exponentially convergent:

$$
V_{n}(\tau) \leqslant V_{n}(0) \exp \left(\frac{\mu}{\nu} \tau\right), \quad \frac{\mu}{\nu}<0
$$

Proof. Taking into account the definition (33a), we have

$$
V_{n}(\tau) \leqslant \nu\left\|\Delta \underline{y}_{a}(\tau)\right\|^{2}, \quad \nu>\phi_{n+1} .
$$

From the inequality 40a, we deduce

$$
\begin{aligned}
\frac{\dot{V}_{n}(\tau)}{V_{n}(\tau)} & \leqslant \frac{\mu\left\|\Delta \underline{y}_{a}(\tau)\right\|^{2}}{V_{n}(\tau)}, \\
\max \left(r_{i i}+q_{i i}\right) & <\mu<0, \quad i=1, \ldots, n+1 .
\end{aligned}
$$

Bounding (44a) with (43) gives

$$
\frac{\dot{V}_{n}(\tau)}{V_{n}(\tau)} \leqslant \frac{\mu\left\|\Delta \underline{y}_{a}(\tau)\right\|^{2}}{\nu\left\|\Delta \underline{y}_{a}(\tau)\right\|^{2}}
$$

which is reduced to

$$
\frac{\mathrm{d} V_{n}(\tau)}{V_{n}(\tau)} \leqslant \frac{\mu}{\nu} \mathrm{d} \tau
$$

We deduce (42) through integration, which indicates the exponential convergence.

2.4. Synthesis of observer parameters. We wish to perform a synthesis of the gains $\underline{h}_{a}$ of (30f) guaranteeing the convergence of the observer. To do this, we consider the term $\Delta \underline{\tilde{\Psi}}(\tau)$ of $(30$ a as the perturbing input of a linear system with constant parameters of the transfer function $1 / D(s)$. The transfer function in the Laplace domain of (30a) is written as

$$
\begin{aligned}
\frac{\Delta y_{1}(s)}{s \Delta \widetilde{\Psi}(s)} & =\frac{1}{D(s)}, \\
D(s) & =s^{n+1}+h_{n} s^{n}+\cdots+h_{1} s+h_{0} .
\end{aligned}
$$


We now seek a group of parameters $\underline{h}_{a}$ (30f) which fit the stability conditions (32). Given (29), we note that $\dot{\widetilde{\Psi}}(\tau)$ is directly affected by $\omega_{o}$, and therefore by $\|\dot{\widetilde{\Psi}}(\tau)\|$.

Applying the Lipschitz assumption (31), the constant $L$ is dependent on $\omega_{o}$. $L$ influences the squared conditions in (32). By using $\omega_{0}$ to keep $L$ small, it is possible to ensure that the stability conditions remain independent of the system's time-scale, and to arbitrarily define the gains $h_{i}$. One simple way to do this is to choose a polynomial with multiple poles:

$$
\begin{aligned}
D(s) & =(s+\nu)^{n+1} \\
& =\sum_{i=0}^{n+1} h_{k} s^{k}, \quad k=n+1-i, \\
h_{k} & =\nu^{i} \frac{(n+1) !}{i ! k !}
\end{aligned}
$$

The value of the pole $0<\nu \leqslant 1$ allows us to somewhat weight the binomial coefficients 48c and to approach the limiting stability conditions. Anyway, the pulse choice $\omega_{o}$ is predominant for the observer function. If the gains thus obtained satisfy the conditions in (32), then the response of the observer converges towards that of the system. The group of parameters is uniquely determined by the order $n$, and the speed of observer convergence is defined by the choice pulse $\omega_{o}$ and the multiple pole $\nu$.

\section{Simulations}

To illustrate Section 2, we propose two different examples: a non-linear Düffing system (Gille et al., 1988) (Section 3.1), a mass-spring system and a Lorenz strange attractor (Section 3.2), used as a model in meterology to predict the convection of air masses (Lorenz, 1963).

3.1. Düffing system. It is written in controller canonical form as follows:

$$
\begin{aligned}
\dot{x}_{2}(t) & =\Psi[\underline{x}(t), \underline{u}(t)], \\
\dot{x}_{1}(t) & =x_{2}(t), \\
y(t)= & x_{1}(t)+\eta(t), \quad n=2, \\
\Psi[\underline{x}(t), \underline{u}(t)]= & -a_{1} \ell_{1}(t) x_{1}(t)-a_{2} x_{2}(t) \\
& +b_{1} u_{11}(t)+b_{2} u_{12}(t), \\
\ell_{1}(t)= & 1+a_{3} x_{1}(t)^{2}, \\
\underline{x}(t)= & {\left[\begin{array}{ll}
x_{1}(t) & x_{2}(t)
\end{array}\right], } \\
\underline{u}(t)= & {\left[\begin{array}{ll}
u_{11}(t) & u_{12}(t)
\end{array}\right], }
\end{aligned}
$$

with the parameters

$$
\begin{aligned}
& a_{1}=157.91, \quad a_{2}=2 \pi, \quad a_{3}=0.4, \\
& b_{1}=100, \quad b_{2}=10 .
\end{aligned}
$$

The measured output $y(t)$ is the juxtaposition of the system output $x_{1}(t)$ of the Düffing and a white noise with limited bandwidth $\eta(t)$. The signal-to-noise ratio is 10 . The input $u_{11}(t)$, subsequently exploitable by the observer, is defined in terms of

$$
u_{11}(t)= \begin{cases}1, & 0 \leqslant t<2 s \\ 0, & t \geqslant 2 s\end{cases}
$$

The input $u_{12}(t)$ is white noise with a limited bandwidth amplitude of \pm 1 , considered non-measurable, which will produce a correlated noise on the vector $\underline{x}(t)$ of the system. The normalized representation of (49) is written as follows:

$$
\begin{aligned}
& \dot{x}_{2}(\tau)=\widetilde{\Psi}[\underline{x}(\tau), \underline{u}(\tau)], \\
& \dot{x}_{1}(\tau)=x_{2}(\tau) \text {, } \\
& y(\tau)=x_{1}(\tau), \quad f_{o}=\frac{1}{T_{o}}=4 \mathrm{~Hz}, \\
& \widetilde{\Psi}[\underline{x}(\tau), \underline{u}(\tau)]=-\frac{a_{1}}{\omega_{o}^{2}} \ell_{1}(\tau) x_{1}(\tau)-\frac{a_{2}}{\omega_{o}} x_{2}(\tau) \\
& +\frac{b_{1}}{\omega_{o}^{2}} u_{11}(\tau)+\frac{b_{2}}{\omega_{o}^{2}} u_{12}(\tau), \\
& \ell_{1}(\tau)=1+a_{3} x_{1}(\tau)^{2}, \\
& \underline{x}(\tau)=\left[\begin{array}{ll}
x_{1}(\tau) & x_{2}(\tau)
\end{array}\right], \\
& \underline{u}(\tau)=\left[\begin{array}{ll}
u_{11}(\tau) & u_{12}(\tau)
\end{array}\right] .
\end{aligned}
$$

The observer (11) applied to 52 is written as follows:

$$
\begin{aligned}
\dot{\hat{x}}_{1}(\tau)= & \breve{x}_{2}(\tau)+h_{2} \Delta y_{1}(\tau), \\
\dot{\breve{x}}_{2}(\tau)= & I_{0}(\tau)+h_{1} \Delta y_{1}(\tau) \\
& +\widetilde{\Psi}\left[\underline{v}(\tau), u_{11}(t)\right], \\
\dot{I}_{0}(\tau)= & h_{0} \Delta y_{1}(\tau), \\
\Delta y_{1}(\tau)= & y(\tau)-\widehat{x}_{1}(\tau), \\
\widetilde{\Psi}\left[\underline{v}(\tau), u_{11}(\tau)\right]= & -\frac{a_{1}}{\omega_{o}^{2}} \widehat{\ell}_{1}(\tau) \widehat{x}_{1}(\tau), \\
& -\frac{a_{2}}{\omega_{o}} \breve{x}_{2}(\tau)+\frac{b_{1}}{\omega_{o}^{2}} u_{11}(\tau), \\
\hat{\ell}_{1}(\tau)= & 1+a_{3} \widehat{x}_{1}(\tau)^{2} \\
\underline{v}(\tau)= & {\left[\breve{x}_{2}(\tau) \quad \widehat{x}_{1}(\tau)\right] }
\end{aligned}
$$

The initial conditions of the system are $x_{1}(0)=x_{2}(0)=$ 0 . Those of the observer are $I_{0}(0)=\breve{x}_{2}(0)=0, \widehat{x}_{1}(0)=$ 1. Using $\widetilde{\Psi}[\underline{x}(\tau), \underline{u}(\tau)] \underline{52 \mathrm{~d}}$ and $\widetilde{\Psi}\left[\underline{v}(0), u_{11}(0)\right]$ 53e, it is possible to form $\Delta \dot{\widetilde{\Psi}}(\tau)$ (29) and to calculate its temporal derivative. Using (52) and (53), we determine $\Delta \underline{y}_{a}(\tau)^{T}=\underline{x}_{a}(\tau)^{T}-\underline{\widetilde{x}}_{a}(0)^{T}$ (27), (28). All this will allow calculating the ratio $|\dot{\widetilde{\Psi}}(\tau)| /\left\|\underline{x}_{a}(\tau)\right\|$, illustrated in Fig. 2(a), and to set the Lipschitz constant $L=0.3$. 


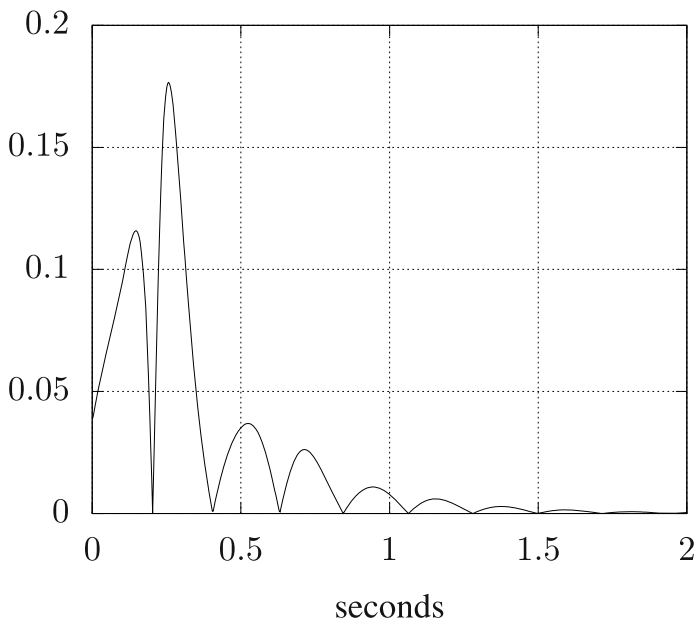

(a)

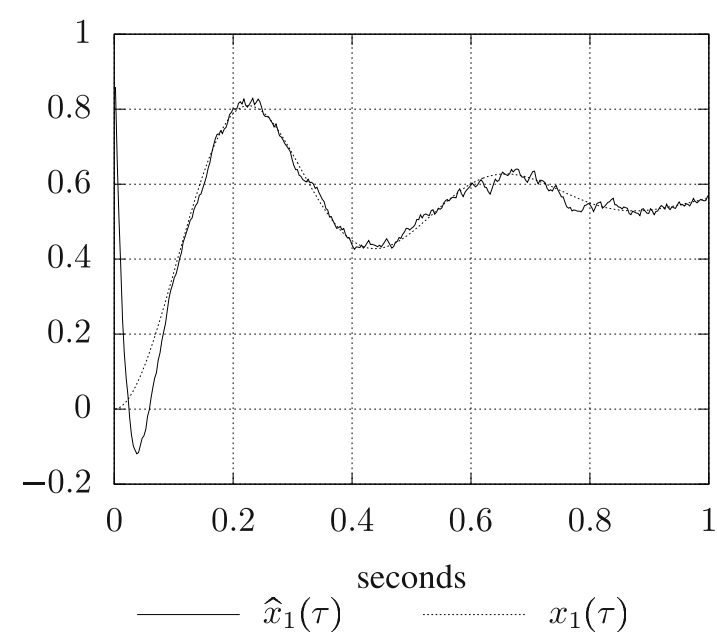

(c)

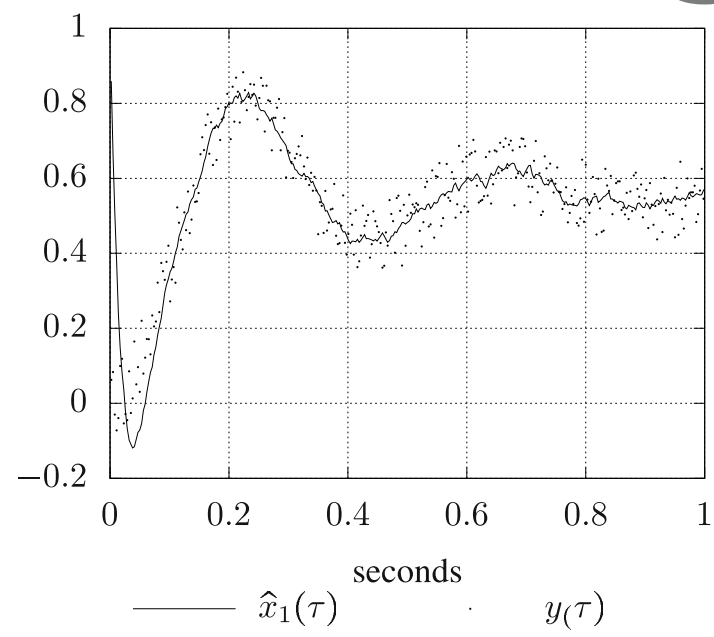

(b)

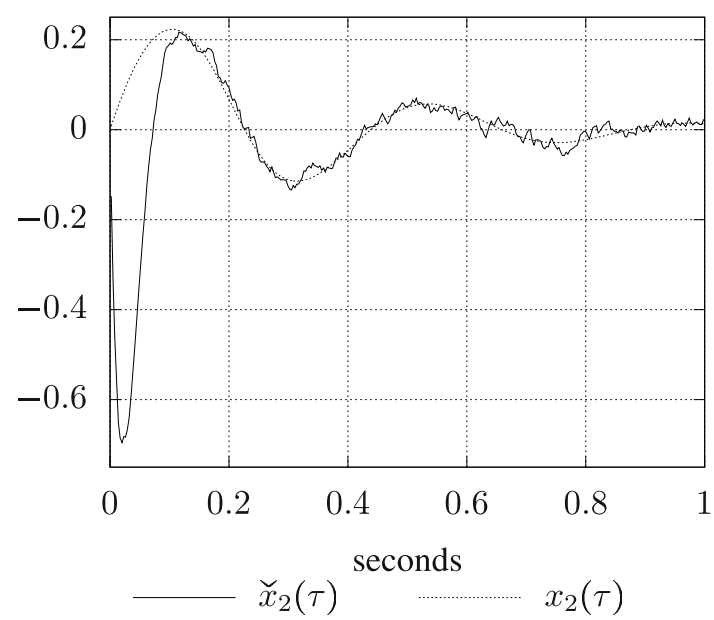

(d)

Fig. 2. Observation of a Düffing system: $|\dot{\widetilde{\Psi}}(\tau)| /\left\|\underline{x}_{a}(\tau)\right\|$ (a), observer response $\widehat{x}_{1}(\tau)$ (part 1) (b), observer response $\widehat{x}_{1}(\tau)$ (part 2) (c), observer reconstruction of $x_{2}(\tau)(\mathrm{d})$.

Setting parameters $\lambda=1 / 8, \sigma=1$ and $\phi_{3}=2$ (32c) and using (41), we obtain $\phi_{2}=2, \phi_{1}=4$, and get the matrix $\underline{P}$ (33). The limiting conditions (32) for observer stability are given by

$$
h_{0} \geqslant 0.427, \quad h_{1} \geqslant 0.403, \quad h_{2} \geqslant 1.03 \text {. }
$$

Using (48) with $n=2, \nu=1$, the gains $h_{i}$ are set as follows:

$$
\underline{h}_{a}^{T}=\left[\begin{array}{lll}
1 & 3 & 3
\end{array}\right] .
$$

This set of parameters satisfies the conditions in (54).

Figures 2 (b) and (c) show the observer response $\hat{x}_{1}(\tau)$ to the measured output $y(t)$ on the one hand, and to the theoretical system response $x_{1}(\tau)$ on the other, which is free of noise $\left(u_{12}(\tau)=\eta(\tau)=0\right)$. Figure 2(d) illustrates the response of the variable $\breve{x}_{2}(\tau)$ to the theoretical system response $x_{2}(\tau)$, which is free of noise. These observations converge nicely and prove robust to measurement noise.

3.2. Lorenz strange attractor. To complement Section 2 consider a model of convection of air masses, used in meteorology (Lorenz, 1963), described by the state representation:

$$
\begin{aligned}
\dot{z}_{x}(t) & =\sigma z_{y}(t)-\sigma z_{x}(t) \\
\dot{z}_{y}(t) & =-z_{y}(t)+\rho z_{x}(t)-z_{x}(t) z_{z}(t), \\
\dot{z}_{z}(t) & =-\beta z_{z}(t)+z_{x}(t) z_{y}(t) \\
y(t) & =z_{x}(t) \\
\underline{z}(t) & =\left[z_{x}(t), z_{y}(t), z_{z}(t)\right]
\end{aligned}
$$

We choose parameters and the following initial 
conditions:

$$
\begin{array}{r}
\beta=1, \quad \sigma=4, \quad \rho=20 \\
z_{x}(0)=z_{y}(0)=z_{z}(0)=10,
\end{array}
$$

as has been proposed for the first strange attractor which describes chaotic oscillations. Its representation transformed in the canonical form of regulation is the following:

$$
\begin{aligned}
\dot{x}_{3}(t)= & \Psi \underline{x}(t)] \\
\Psi[\underline{x}(t)]= & -(1+\sigma+\beta) x_{3}(t)-\beta(1+\sigma) x_{2}(t) \\
& +\beta \sigma(\rho-1) x_{1}(t)-x_{1}(t)^{2} x_{2}(t) \\
& -\sigma x_{1}(t)^{3} \\
& +\frac{x_{2}(t) x_{3}(t)+(1+\sigma) x_{2}(t)^{2}}{x_{1}(t)} \\
\dot{x}_{2}(t)= & x_{3}(t) \\
\dot{x}_{1}(t)= & x_{2}(t), \\
y(t)= & x_{1}(t), \quad n=3
\end{aligned}
$$

with (58b) as the non-linear $\Psi$ function of the system. The latter may or may not be dependent on $\underline{U}$. The only constraint to fulfill is the existence of a Lipschitz type distance $\Delta \dot{\widetilde{\Psi}}(31)$. The transformation system (58) linking to the initial state is given by

$$
\begin{aligned}
& z_{x}(t)=x_{1}(t) \\
& z_{y}(t)=x_{1}(t)+\left(\frac{1}{\sigma}\right) x_{2}(t) \\
& z_{z}(t)=\rho-1-\left(1+\frac{1}{\sigma}\right) \frac{x_{2}(t)}{x_{1}(t)}-\left(\frac{1}{\sigma}\right) \frac{x_{3}(t)}{x_{1}(t)}
\end{aligned}
$$

Figure 3(a) illustrates its well known behaviour, in the plane $z_{x} z_{z}$. Parameterized in this way, it possesses its characteristic natural frequency around $1 \mathrm{~Hz}$. It is interesting to note that $x_{1}(t)$ passes near zero during a few brief transitions, making observation possible with a function $\widetilde{\Psi}$ such as (58b). The normalized notation of (58) is the following:

$$
\begin{aligned}
\dot{x}_{3}(\tau)= & \widetilde{\Psi}[\underline{x}(\tau)] \\
\widetilde{\Psi}[\underline{x}(\tau)]= & -\frac{1+\sigma+\beta}{\omega_{o}} x_{3}(\tau)-\frac{\beta(1+\sigma)}{\omega_{o}^{2}} x_{2}(\tau) \\
& +\frac{\beta \sigma(\rho-1)}{\omega_{o}^{3}} x_{1}(\tau)-\frac{x_{1}(\tau)^{2} x_{2}(\tau)}{\omega_{o}^{2}} \\
& -\frac{\sigma x_{1}(\tau)^{3}}{\omega_{o}^{3}}
\end{aligned}
$$

$$
\begin{aligned}
& +\frac{x_{2}(\tau) x_{3}(\tau)+\frac{1+\sigma}{\omega_{o}} x_{2}(\tau)^{2}}{x_{1}(\tau)}, \\
\dot{x}_{2}(\tau)= & x_{3}(\tau), \\
\dot{x}_{1}(\tau)= & x_{2}(\tau), \\
y(\tau)= & x_{1}(\tau), \quad f_{o}=\frac{1}{T_{o}}=2.5 \mathrm{~Hz} .
\end{aligned}
$$

It is evident that the previous type of observer (Schwaller et al., 2013) cannot be used in this case, because the function $\widetilde{\Psi}$ is not decomposable into the form $\widetilde{\Psi}[\underline{x}(\tau)]=f_{1}\left(x_{1}(\tau), x_{2}(\tau), \underline{u}(\tau)\right)+\dot{f}_{2}\left(x_{1}(\tau), x_{2}(\tau)\right)$, as has been proposed. The system of normalized transformation linking (60) the initial state space is given by

$$
\begin{aligned}
z_{x}(\tau)= & x_{1}(\tau) \\
z_{y}(\tau)= & x_{1}(\tau)+\left(\frac{\omega_{o}}{\sigma}\right) x_{2}(\tau) \\
z_{z}(\tau)= & \rho-1-\left(\omega_{o}+\frac{\omega_{o}}{\sigma}\right) \frac{x_{2}(\tau)}{x_{1}(\tau)} \\
& -\left(\frac{\omega_{o}^{2}}{\sigma}\right) \frac{x_{3}(\tau)}{x_{1}(\tau)}
\end{aligned}
$$

Inverting a1 and knowing $z_{x}(\tau), z_{x}(\tau), z_{z}(\tau)$ (56), it is possible to reconstruct $\underline{x}_{a}(\tau)$. Calculating the numerical derivation of $\widetilde{\Psi}(\tau)$ 60b , it is subsequently possible to determine the most pessimistic Lipschitz constant $L=0.15$ (Fig. 3 (b)) after $40 \mathrm{~s}$ of the test. The observer structure, illustrated by Fig. 11 (11), takes the following form:

$$
\begin{aligned}
\dot{\widehat{x}}_{1}(\tau)= & \widehat{x}_{2}(\tau)+h_{3} \Delta y_{1}(\tau), \\
\dot{\widehat{x}}_{2}(\tau)= & \breve{x}_{3}(\tau)+h_{2} \Delta y_{1}(\tau), \\
\dot{\check{x}}_{2}(\tau)= & \breve{x}_{3}(\tau), \\
\dot{\check{x}}_{3}(\tau)= & I_{0}(\tau)+h_{1} \Delta y_{1}(\tau) \\
& +\widetilde{\Psi}\left[\underline{x}(\tau), \widehat{x}_{1}(\tau)\right], \\
\dot{I}_{0}(\tau)= & h_{0} \Delta y_{1}(\tau), \\
\Delta y_{1}(\tau)= & y(\tau)-\widehat{x}_{1}(\tau),
\end{aligned}
$$

with the function

$$
\begin{aligned}
\widetilde{\Psi}\left[\underline{\breve{x}}(\tau), \widehat{x}_{1}(\tau)\right] & \\
= & -\frac{1+\sigma+\beta}{\omega_{o}} \breve{x}_{3}(\tau)-\frac{\beta(1+\sigma)}{\omega_{o}^{2}} \breve{x}_{2}(\tau) \\
& +\frac{\beta \sigma(\rho-1)}{\omega_{o}^{3}} \widehat{x}_{1}(\tau)-\frac{\widehat{x}_{1}(\tau)^{2} \breve{x}_{2}(\tau)}{\omega_{o}^{2}} \\
& -\frac{\sigma \widehat{x}_{1}(\tau)^{3}}{\omega_{o}^{3}} \\
& +\frac{\breve{x}_{2}(\tau) \breve{x}_{3}(\tau)+\frac{1+\sigma}{\omega_{o}} \breve{x}_{2}(\tau)^{2}}{\widehat{x}_{1}(\tau)} .
\end{aligned}
$$




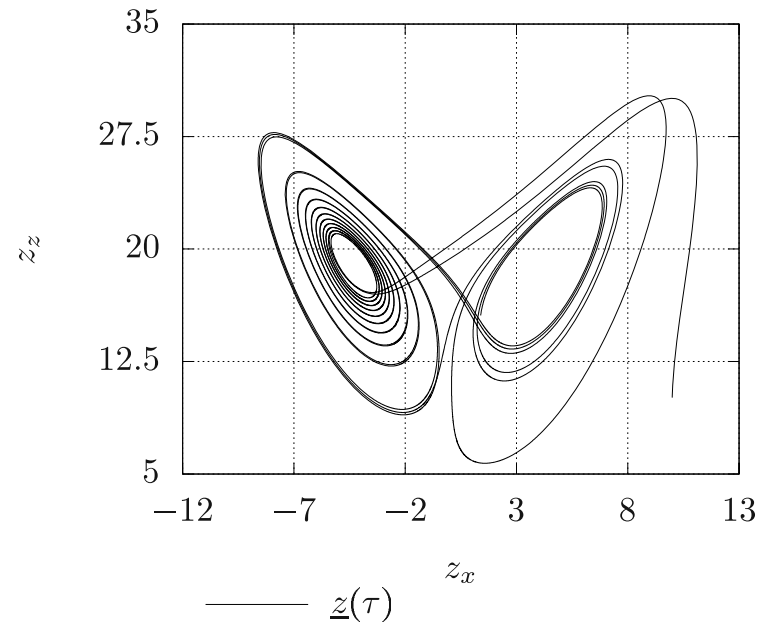

(a)

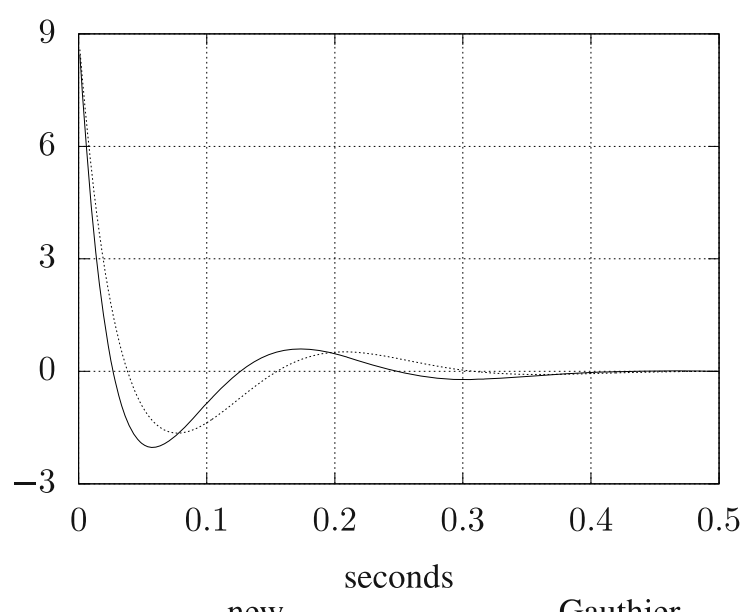

(c)

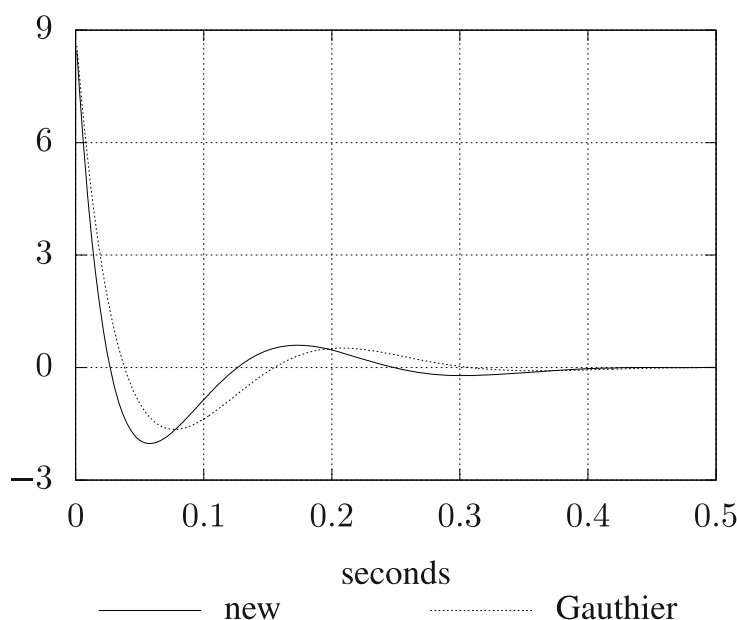

(e)

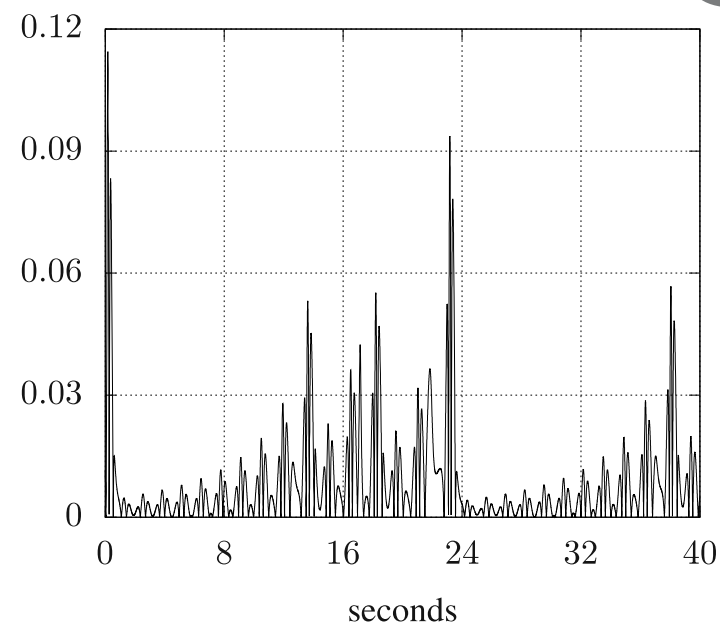

(b)

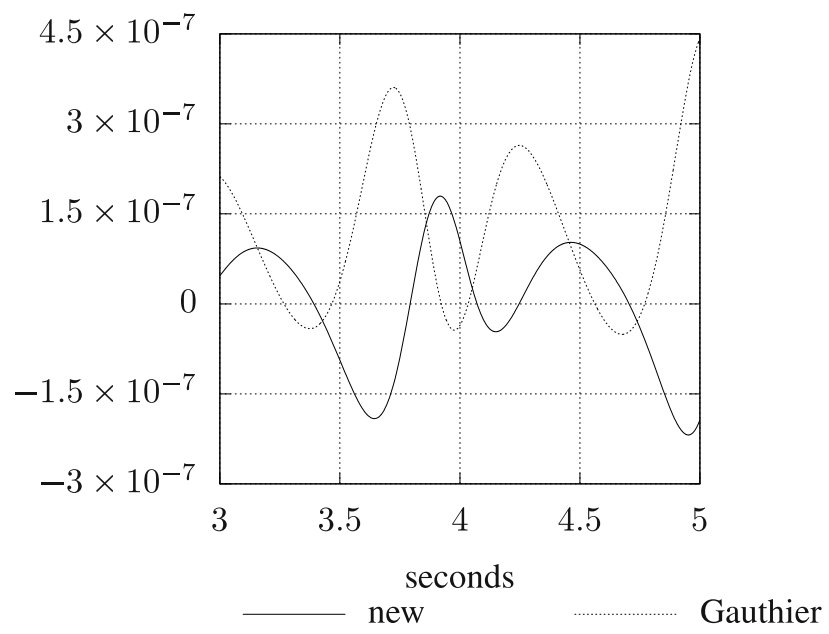

(d)

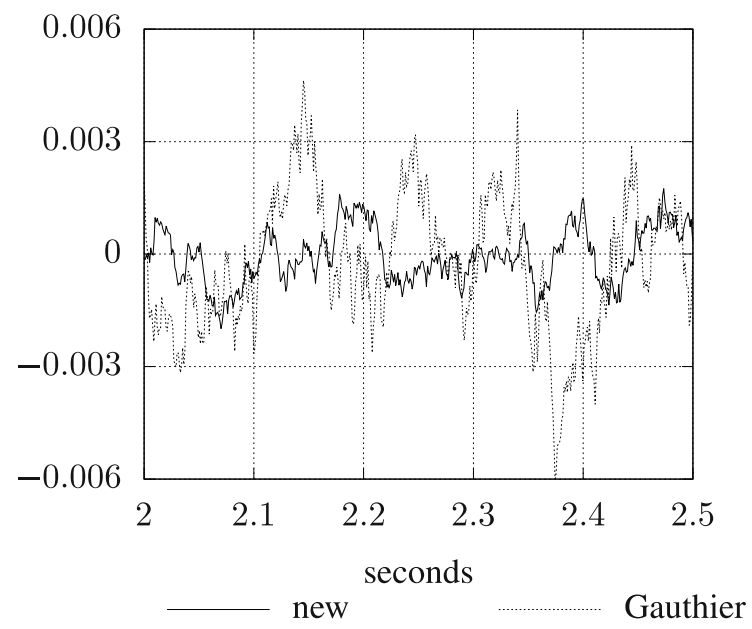

(f)

Fig. 3. Observation of a Lorenz strange attractor, part 1: state trajectory (a), $|\dot{\widetilde{\Psi}}(\tau)| /|| \underline{x}_{a}(\tau)||$ (b), $\Delta y(t)$, asymptotic convergence (c), $\Delta y(t)$, stabilised convergence (d), $\Delta y(\tau)$, asymptotic convergence (e), $\Delta y(\tau)$, stabilized convergence (f). 


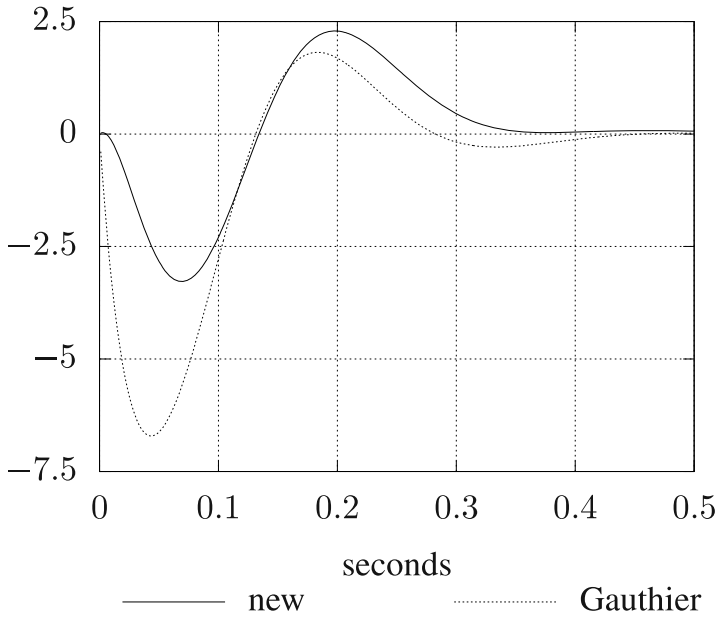

(a)

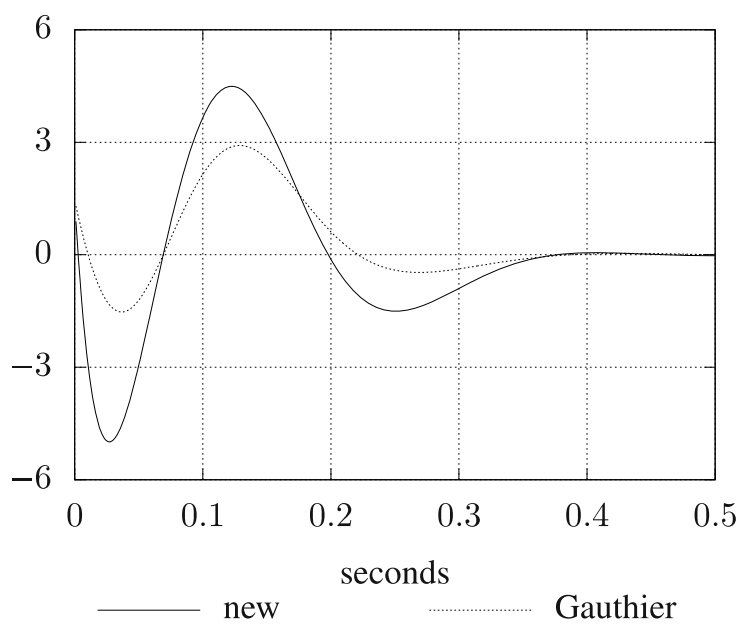

(c)

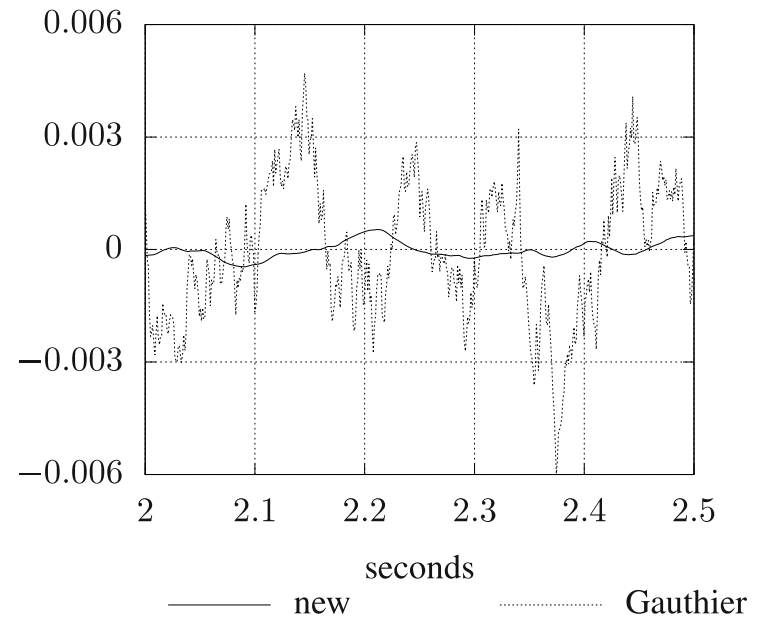

(b)

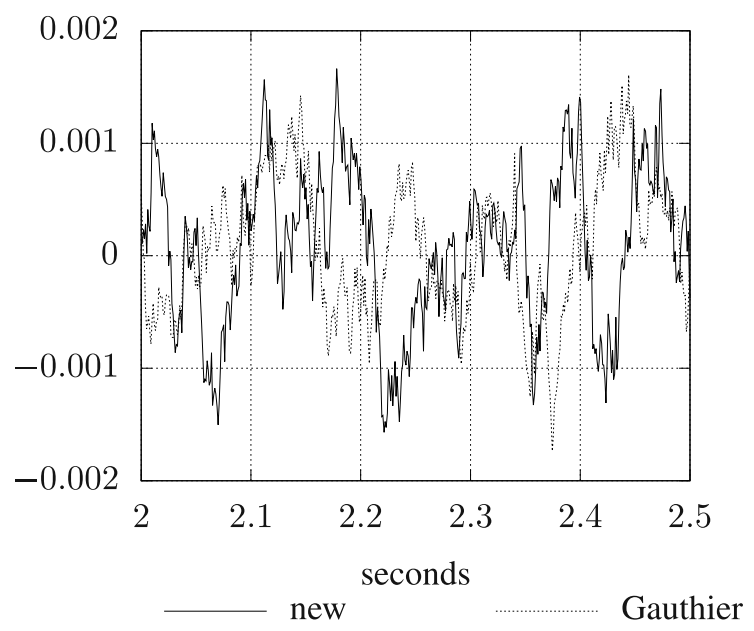

(d)

Fig. 4. Observation of a Lorenz strange attractor, part 2: $\Delta \widehat{x}_{2}(\tau), \Delta \breve{x}_{2}(\tau)$, asymptotic convergence (a), $\Delta \widehat{x}_{2}(\tau), \Delta \breve{x}_{2}(\tau)$, stabilized convergence (b), $\Delta \widehat{x}_{3}(\tau), \Delta \check{x}_{3}(\tau)$, asymptotic convergence (c), $\Delta \widehat{x}_{3}(\tau), \Delta \check{x}_{3}(\tau)$, stabilized convergence (d).

The transformation system linking the observer to the initial estimated space is the following:

$$
\begin{aligned}
\widehat{z}_{x}(\tau)= & \widehat{x}_{1}(\tau), \\
\widehat{z}_{y}(\tau)= & \widehat{x}_{1}(\tau)+\left(\frac{\omega_{o}}{\sigma}\right) \breve{x}_{2}(\tau), \\
\widehat{z}_{z}(\tau)= & \rho-1-\left(\omega_{o}+\frac{\omega_{o}}{\sigma}\right) \frac{\widehat{x}_{2}(\tau)}{\widehat{x}_{1}(\tau)} \\
& -\left(\frac{\omega_{o}^{2}}{\sigma}\right) \frac{\breve{x}_{3}(\tau)}{\widehat{x}_{1}(\tau)} .
\end{aligned}
$$

If one fixes the parameter $\phi_{4}=2$, using (41) one can obtain $\phi_{3}=2, \phi_{2}=4, \phi_{1}=8$. We choose $\lambda=1 / 8$ and thus define the matrix $\underline{P}$ 33b). Using the stability conditions (32), we obtain the limiting conditions that must be satisfied to synthesize the gains $h_{i}$ :

$$
\begin{aligned}
& h_{0} \geqslant 0.171, \quad h_{1} \geqslant 0.273, \\
& h_{2} \geqslant 0.512, \quad h_{3} \geqslant 0.891 .
\end{aligned}
$$

Using (48) for $n=3, \nu=1$, we fix the gains at

$$
\underline{h}_{a}^{T}=\left[\begin{array}{llll}
1 & 4 & 6 & 4
\end{array}\right],
$$

for which the values satisfy the conditions (65). The initial observer conditions are fixed as $\underline{x}(0)=\underline{0}, \hat{x}_{1}(0)=$ $1, \hat{x}_{2}(0)=0$. Figures 3 (c) and 3 (d) illustrate the stability of the observer convergence in the transitory phase, compared with that of a Gauthier observer. The responses are very similar. The next test was performed in a noisy situation, by adding to $x_{1}(\tau)$ white noise with a passband with a limited amplitude of 0.2 . For the observer (11), we visualise the following errors:

$$
\Delta \breve{x}_{i}(\tau)=x_{i}(\tau)-\breve{x}_{i}(\tau), \quad i=2, \ldots, n .
$$


The same is for the Gauthier observer-we visualise

$$
\Delta \widehat{x}_{i}(\tau)=x_{i}(\tau)-\widehat{x}_{i}(\tau), \quad i=2, \ldots, n
$$

For the same initial conditions as for the trial without noise, Figs. 3(e), 3(f), 4(a), 4(b), 4(c), 4(d) illustrate the results obtained from noisy measurements $y(\tau)$. Systematically, the state distances are lower for the observer (11) than for the Gauthier observer except for state variables of order $n$, which are comparable. The observer 111 is thus more robust to noise and provides the best estimates of variables $z_{y}(\tau), z_{z}(\tau)$ across the transformation 61).

\section{Conclusions and perspectives}

The results obtained in this study considerably extend the field of application compared with the previous one, as much for the type of non-linearities that it can treat as for the limiting stability conditions. The proposed observer structure allows expression of the convergence dynamics in the form of a non-linear differential equation with constant coefficients. Its stability is demonstrated when the non-linearities are at least locally of the Lipschitz type. In this case, $n$ inequalities are determined by a quadratic Lyapunov function, which guarantees the stability. One thus demonstrates the exponentially convergent character of the estimates.

Gain synthesis in the scaled space provides gains independently of the time scale of the physical system and of the observer, not relying on the order $n$ of the differential equations. Decoupling error corrections and the state space used to reconstruct the non-linear function $\Psi(t)$ strongly reinforces the robustness of estimates to measurement noise compared with results usually obtained with high gain observers.

The strategy PI permits modelling external perturbations and assures a unit gain in the observation dynamics. This constitutes an advantage which can be exploited by a regulation stage.

The reconstructed state vector has the major advantage of being exact and directly exploitable for state control without any additional transformations.

Further simulations under conditions of parametric uncertainty, minimized by online parameter identification, may help increase the robustness of such estimates, even in the presence of instrumental noise and external system perturbation. This can ultimately be extended to MIMO systems.

\section{References}

Alma, M. and Darouach, M. (2014). Adaptive observers design for a class of linear descriptor systems, Automatica 50(2): 578-583.
Bestle, D. and Zeitz, M. (1983). Canonical form observer design for non-linear time-variable systems, International Journal of Control 38(2): 419-431.

Bezzaoucha, S., Marx, B., Maquin, D. and Ragot, J. (2013). State and parameter estimation for time-varying systems: A Takagi-Sugeno approach, American Control Conference (ACC), Washington, DC, USA, pp. 1050-1055.

Bodizs, L., Srinivasan, B. and Bonvin, D. (2011). On the design of integral observers for unbiased output estimation in the presence of uncertainty, Journal of Process Control 21(3): 379-390.

Boker, A. and Khalil, H. (2013). Nonlinear observers comprising high-gain observers and extended Kalman filters, Automatica 49(12): 3583-3590.

Bouraoui, I., Farza, M., Ménard, T., Abdennour, R.B., M'Saad, M. and Mosrati, H. (2015). Observer design for a class of uncertain nonlinear systems with sampled outputs: Application to the estimation of kinetic rates in bioreactors, Automatica 55: 78-87.

Chen, W., Khan, A.Q., Abid, M. and Ding, S.X. (2011). Integrated design of observer based fault detection for a class of uncertain nonlinear systems, International Journal of Applied Mathematics and Computer Science 21(3): 423-430, DOI: 10.2478/v10006-011-0031-0.

Ciccarella, G., Mora, M.D. and Germani, A. (1993). A Luenberger-like observer for nonlinear systems, International Journal of Control 57(3): 537-556.

Efimov, D. and Fridman, L. (2011). Global sliding-mode observer with adjusted gains for locally Lipschitz systems, Automatica 47(3): 565-570.

Farza, M., Bouraoui, I., Ménard, T., Abdennour, R.B. and M'Saad, M. (2014). Adaptive observers for a class of uniformly observable systems with nonlinear parametrization and sampled outputs, Automatica 50(11): 2951-2960.

Farza, M., M'Saad, M., Triki, M. and Maatoug, T. (2011). High gain observer for a class of non-triangular systems, Systems and Control Letters 60(1): 27-35.

Fliess, M. (1990). Generalized controller canonical forms for linear and nonlinear dynamics, IEEE Transactions on Automatic Control 35(9): 994-1001.

Gauthier, J. and Bornard, G. (1981). Observability for any $u(t)$ of a class of nonlinear systems, IEEE Transactions on Automatic Control AC-26(4): 922-926.

Gauthier, J., Hammouri, H. and Othman, S. (1992). A simple observer for nonlinear systems. Applications to bioreactors, IEEE Transactions on Automatic Control 37(6): 875-880.

Ghosh, D., Saha, P. and Chowdhury, A. (2010). Linear observer based projective synchronization in delay Roessler system, Communications in Nonlinear Science and Numerical Simulation 15(6): 1640-1647.

Gille, J., Decaulne, P. and Pélegrin, M. (1988). Systèmes asservis non linéaires, 5 ième edn, Dunod, Paris. 
Gißler, J. and Schmid, M. (1990). Vom Prozeß zur Regelung. Analyse, Entwurf, Realisierung in der Praxis, Siemens, Berlin/München.

Glumineau, A. and Lôpez-Morales, V. (1999). Transformation to State Affine System and Observer Design, Lecture Notes in Control and Information Science, Vol. 244, Springer, London.

Guerra, T., Estrada-Manzo, V. and Lendek, Z. (2015). Observer design for Takagi-Sugeno descriptor models: An LMI approach, Automatica 52: 154-159.

Hermann, R. and Krener, A. (1977). Nonlinear controllability and observability, IEEE Transactions on Automatic Control AC-22(5): 728-740.

Krener, A. and Isidori, A. (1983). Linearization by output injection and nonlinear observers, Systems \& Control Letters 3(1): 47-52.

Lorenz, E. (1963). Deterministic nonperiodic flow, Journal of the Atmospheric Sciences 20(2): 130-141.

Luenberger, D. (1966). Observers for multivariable systems, IEEE Transactions on Automatic Control AC-11(2): 190-197.

Martínez-Guerra, R., Mata-Machuca, J., Aguilar-López, R. and Rodríguez-Bollain, A. (2011). Applications of Chaos and Nonlinear Dynamics in Engineering, Vol. 1, Springer-Verlag, Berlin/Heidelberg.

Mazenc, F. and Dinh, T. (2014). Construction of interval observers for continuous-time systems with discrete measurements, Automatica 50(10): 2555-2560.

Menini, L. and Tornambè, A. (2011). Design of state detectors for nonlinear systems using symmetries and semi-invariants, Systems and Control Letters 60(2): 128-137.

Mobki, H., Sadeghia, M. and Rezazadehb, G. (2015). Design of direct exponential observers for fault detection of nonlinear MEMS tunable capacitor, IJE Transactions A: Basics 28(4): 634-641.

Morales, A. and Ramirez, J. (2002). A PI observer for a class of nonlinear oscillators, Physics Letters A 297(3-4): 205-209.

Raghavan, S. and Hedrick, J. (1994). Observer design for a class of nonlinear systems, International Journal of Control 59(2): 515-528.

Rauh, A., Butt, S.S. and Aschemann, H. (2013). Nonlinear state observers and extended Kalman filters for battery systems, International Journal of Applied Mathematics and Computer Science 23(3): 539-556, DOI: 10.2478/amcs-2013-0041.

Röbenack, K. and Lynch, A. (2004). An efficient method for observer design with approximately linear error dynamics, International Journal of Control 77(7): 607-612.

Röbenack, K. and Lynch, A.F. (2006). Observer design using a partial nonlinear observer canonical form, International Journal of Applied Mathematics and Computer Science 16(3): 333-343.
Schwaller, B., Ensminger, D., Dresp-Langley, B. and Ragot, J. (2013). State estimation for a class of nonlinear systems, International Journal of Applied Mathematics and Computer Science 23(2): 383-394, DOI: 10.2478/amcs-2013-0029.

Söffker, D., Yu, T. and Müller, P. (1995). State estimation of dynamical systems with nonlinearities by using proportional-integral observers, International Journal of Systems Science 26(9): 1571-1582.

Thabet, R., Raïssi, T., Combastel, C., Efimov, D. and Zolghadri, A. (2014). An effective method to interval observer design for time-varying systems, Automatica 50(10): 2677-2684.

Tornambè, A. (1992). High-gain observers for non-linear systems, International Journal of Systems Science 23(9): 1475-1489.

Tyukina, I., Steurb, E., Nijmeijerc, H. and van Leeuwenb, C. (2013). Adaptive observers and parameter estimation for a class of systems nonlinear in the parameters, Automatica 49(8): 24092423.

Veluvolu, K., Soh, Y. and Cao, W. (2007). Robust observer with sliding mode estimation for nonlinear uncertain systems, IET Control Theory and Applications 1(5): 15331540.

Zeitz, M. (1985). Canonical forms for nonlinear-systems, in B. Jakubczyk et al. (Eds.), Proceedings of the Conference on Geometric Theory of Nonlinear Control Systems, Wrocław Technical University Press, Wrocław, pp. 255-278.

Zeitz, M. (1987). The extended Luenberger observer for nonlinear systems, Systems and Control Letters Archive 9(2): 149-156.

Zheng, G., Boutat, D. and Barbot, J. (2009). Multi-output dependent observability normal form, Nonlinear Analysis. Theory, Methods and Applications 70(1): 404-418.

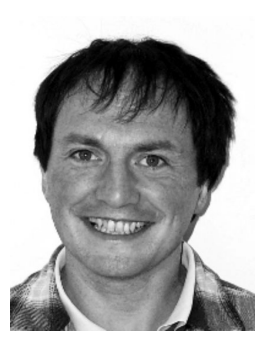

Benoît Schwaller received his Ph.D. in automatic control and biomechanics in 1993 from the Louis Pasteur University of Strasbourg (France). Since 1999, he has been teaching at the University Institute of Technology of Schiltigheim, University of Strasbourg. He conducts his research at INSA Strasbourg, Graduate School of Science and Technology. His fields of interest include real time control, non-linear observer modelling and identification.

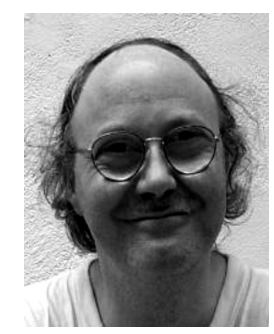

Denis Ensminger obtained a Master's degree in computer science and a Ph.D. in informatics and biomechanics at the Louis Pasteur University of Strasbourg (France). He has worked as an engineer for companies such as Intertechnique or Siemens. Since 1992, he has been a research scientist in computer science at the University Institute of Technology (Strasbourg University). His research at INSA Strasbourg (LGECO), Graduate School of Science and Technology, is mainly focused on signal processing, artificial intelligence and embedded systems. He also works for industry as a consultant. 


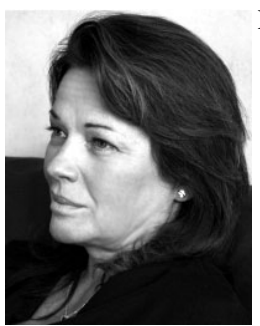

Birgitta Dresp-Langley obtained a Ph.D. in cognitive science in 1992 at University Paris V. She has been a tenured research scientist with CNRS since 1993. Her fields of interest include cognitive systems, perceptual processes, modelling, and abstract expressionist painting.

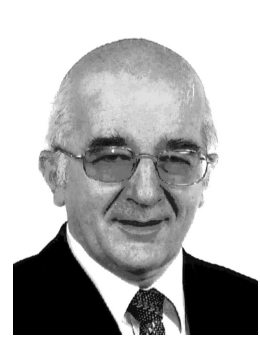

José Ragot received his engineer's degree in 1969 with specialisation in control from ECN Nantes (France), Graduate School of Science and Technology. Then, he joined the University of Nancy (France), where he received his Master's in 1970. In 1973 he obtained his Ph.D. and in 1980 his Diplôme de Doctorat-es-Sciences. Since 1985, José Ragot has been a full professor at the University of Lorraine and a researcher at the Automatic Control Research Center of Nancy (CRAN). His fields of interest include data validation, process diagnosis, fault detection and isolation, modelling and identification.

Received: 19 June 2015

Revised: 15 April 2016

Accepted: 3 June 2016 